\title{
Neuroprotective Effect of Sesamol Against Aluminum Nanoparticles induced Toxicity in Rats
}

Shimaa Abou-Zeid ( $\nabla$ shaimaa.abouzaid@vet.usc.edu.eg )

University of Sadat City https://orcid.org/0000-0001-6804-5967

Basma Elkhadrawey

University of Sadat City

Anis Anis

University of Sadat City

Huda AbuBakr

Cairo University

Badr El-Bialy

University of Sadat City

Hesham Elsabbagh

University of Sadat City

Nermeen El-Borai

University of Sadat City

\section{Research Article}

Keywords: Alumina nanoparticles, sesamol, neurotoxicity, oxidative stress, histopathology

Posted Date: April 5th, 2021

DOI: https://doi.org/10.21203/rs.3.rs-360860/v1

License: @ (i) This work is licensed under a Creative Commons Attribution 4.0 International License. Read Full License 


\section{Abstract}

Alumina nanoparticles (AINPs) are widely used causing neurobehavioral impairment in intoxicated animals and human. Sesamol (SML) emerged as a natural phytochemical with potent antioxidant and anti-inflammatory properties. However, no study has directly tested the potential of SML to protect against AINPs induced detrimental effects on the brain. AINPs $(100 \mathrm{mg} / \mathrm{kg})$ were orally administered to 'rats by gavage with or without oral sesamol $(100 \mathrm{mg} / \mathrm{kg})$ for 28 days. In AINPsintoxicated group, brain AChE activity was elevated. The concentrations of MDA and 8-OHdG were increased suggesting lipid peroxidation and oxidative DNA damage. GSH depletion with inhibited activities of CAT and SOD were demonstrated. Serum levels of IL-1 $\beta$ and IL- 6 were elevated. The expressions of GST, TNF- $\alpha$ and caspase- 3 genes in brain were upregulated. Histopathologically, AINPs induced hemorrhages, edema, neuronal necrosis and/ or apoptosis in medulla oblongata. The cerebellum showed loss of Purkinje cells and the cerebrum showed perivascular edema, neuronal degeneration, necrosis, and neuronal apoptosis. However, concomitant administration of SML with AINPs significantly ameliorated the toxic effects on the brain, reflecting antioxidant, anti-inflammatory and anti-apoptotic effects of SML. Considering these results, sesamol could be promising phytochemical with neuroprotective activity against AINPs-induced neurotoxicity.

\section{Introduction}

The incorporation of nanoparticles into everyday life constitutes an important toxic hazard to human and animals. Alumina (aluminum oxide) nanoparticles (AINPs) account for about $20 \%$ of the global nanoparticle market (Sengul and Asmatulu 2020). They have multiple uses in cosmetics, medicines, electronics, aerospace, chemical engineering, food contact materials, catalysts, ceramics and rubber (Li et al. 2020).

The widespread use of AINPs is associated with possible exposure of human and animals. They may enter the body through food, water, inhalation or dermal contact, where they can readily penetrate biological barriers, due to their small size and high surface reactivity, and accumulate in different organs and tissues (Krause et al. 2020). Moreover, because the cell surface is negatively charged and AINPs are positively charged, these particles are drawn to the interior of cells through electrostatic interactions (Liu et al. 2020a). This in turn produces many toxic effects including hematological changes (De et al. 2020), genotoxicity (Zhang et al. 2017), testicular toxicity (De et al. 2020), carcinogenicity (Dey et al. 2008) immunotoxicity (Li et al. 2020), hepatotoxicity, nephrotoxicity (Morsy et al. 2016) and pulmonary toxicity (Yun et al. 2020).

The CNS was mentioned as a potential target for AINPs. They penetrate the blood-brain barrier (BBB) easily owing to their small size (De et al. 2020) and their effect on BBB permeability through downregulation of the tight junction protein expression such as occludin and claudin-5 (Chen et al. 2008). The penetrating AINPs were reported to accumulate in the hippocampus, olfactory bulb, cerebral cortex, and striatum causing learning and memory dysfunction and depression-like behavior (Shah et al. 2015; Zhang et al. 2021a). Furthermore, AINPs were recognized as a major contributor to many neurodegenerative disorders such as Alzheimer's and Parkinson's diseases (Zhang et al. 2018).

Apart from its essential role in cholinergic neurotransmission, acetylcholinesterase (AChE) has non-cholinergic functions such as its effect on cell survival, neurite outgrowth, and voltage-dependent calcium currents (Whyte and Greenfield 2003). Thus, assessment of this AChE activity is a useful maker of neurotoxicity. Oxidative damage to nerve cell is the first event in a sequence leading to deleterious effects after nanoparticles intoxication (Shah et al. 2015). However, cellular enzymatic and non-enzymatic antioxidants can alleviate this oxidative injury. Thus, determination of MDA and 8-OHdG can be used as valuable markers of oxidative damage to membrane lipids and DNA. In addition, assessment of GSH, SOD, CAT and GST indicates the antioxidant status in brain. 
Apoptosis has been reported as a major pathway of cellular death consequent to nanoparticle evoked oxidative stress (Nogueira et al. 2019; Liu et al. 2020a). As the mitochondria are potential targets for oxidative stress (Manke et al. 2013), cascade reactions occur in the intrinsic (mitochondrial) apoptotic pathway including mitochondrial outer membrane permeabilization (MOMP), and cytochrome c release to the cytoplasm activating caspase- 9 and subsequent caspase-3 (Xiong et al. 2014). Thus, assessment of caspase-3 is a valuable marker for ROS-mediated cell death via mitochondrial pathway.

Nanoparticle-mediated oxidative injury has been shown to orchestrate a series of inflammatory responses (Manke et al. 2013) with release of pro-inflammatory mediators via NF-KB/MAPK activation ( Zhang et al. 2021a). The produced neuroinflammation has been linked to Alzheimer disease and other neurodegenerative disorders (Win-Shwe and Fujimaki 2011). Thus, assessment of the inflammatory cytokines IL-1 $\beta$, IL- 6 and TNF- $\alpha$ evaluates the inflammatory response in intoxicated animals.

Research in the field of toxicology has always focused on offering natural plant oils to alleviate various toxic insults (Abou-Zeid et al. 2018). These oils contain phytochemicals with little harm and strong antioxidant activity making them valuable drugs (Farag et al. 2021). Among them, sesame (Sesamum indicum) oil which contains sesamol (SML), a natural phenolic lignan with the chemical structure 3,4-(Methylenedioxy) phenol (Majdalawieh and Mansour 2019).

Sesamol was reported to be a potent antioxidant with strong scavenging activity against free radicals (Kanimozhi and Prasad 2009). After oral administration, it was readily absorbed and detected in multiple organs due to its unique solubility in both aqueous and oily phases (Jan et al. 2008). In addition, it has a great ability to penetrate the blood-brain barrier due to its high lipophilicity (Ren et al. 2018).

Evidences have been accumulated regarding the therapeutic uses of sesamol specially as antimutagenic and anticancer drug (Majdalawieh and Mansour 2019). Recently, sesamol has been receiving considerable attention as a neuroprotective agent. It alleviated the cognitive deficits and detrimental effects produced in rat models of induced diabetic neuropathy (VanGilder et al. 2009; Chopra et al. 2010), Huntington's disease (Kumar et al. 2010), epilepsy (Hassanzadeh et al. 2014) and chronic intermittent hypoxia (Zhang et al. 2021b) and natural aging model in mouse (Ren et al. 2018, 2020).

We hypothesized that sesamol may exhibit neuroprotective effect towards AINPs induced brain injury. Thus, the present study aimed at investigating the antioxidant, anti-inflammatory and anti-apoptotic potentials of sesamol in AINPsintoxicated rats via assessment of AChE activity, oxidative stress markers, antioxidants, proinflammatory cytokines and caspase-3 expression. In addition, the histopathological alterations in brain were investigated.

\section{Materials And Methods}

\subsection{Chemicals}

Aluminum oxide $\left(\mathrm{Al}_{2} \mathrm{O}_{3}\right.$ ) or alumina nanoparticles (AINPs) (particle sizes $<50 \mathrm{~nm}$ ), and sesamol (SML) were obtained from Sigma-Aldrich (Germany). All other chemicals used were of analytical grade.

\subsection{Characterization of AINPs}

AINPs were characterized as previously mentioned by Li et al. (2020) using transmission electron microscopy (TEM). The diameters of AINPs were analyzed by the Image-Pro Plus software. Suspension of freshly prepared AINPs in bi-distilled water were used after ultrasonic vibration (100 W, $30 \mathrm{kHz}, 30 \mathrm{~min}$ ). In the TEM, a suspension droplet was placed on a $200-$ mesh Cu-lacy lace carbon TEM grid. The film on the TEM grid was dried overnight in a vacuum oven at room temperature $(25 \circ \mathrm{C})$. After water evaporation, the AINPs were dispersed over the TEM grid. TEM measurements were conducted on a JEOL model $2100 \mathrm{~F}$ instrument (Japan) operated at an accelerating voltage of $200 \mathrm{kV}$. 


\subsection{Animals}

All experimental procedures in this study were approved in advance (approval number: VUSC-007-1-20) by the Institutional Animal Care and Use Committee of University of Sadat City, Egypt, and conducted out following National Institutes of Health Guidelines for the Care and Use of Laboratory Animals.

Eighty young adult male Sprague-Dawley rats (140-160 g) were obtained from the Laboratory Animal Farm, Faculty of Veterinary Medicine, University of Sadat City, Egypt, and were acclimated in our facility for one week, prior to the experiment. Animals were caged at standardized conditions ( $12 \mathrm{~h} \mathrm{light/} \mathrm{dark} \mathrm{period;} \mathrm{temperature} 23 \pm 2^{\circ} \mathrm{C}$; humidity $50 \%$ ) and allowed free access to food and distilled water during the acclimatization period and throughout the experiment. Animals were randomly allocated into four groups, 20 rats each.

\subsection{Experimental design}

All experimental groups received the treatments daily by stomach tube for 28 days. Group I (Control): received distilled water. Group II (SML group): received SML at a dose level of $100 \mathrm{mg} / \mathrm{kg} /$ day. Group III (AINPs group): received AINPs at $100 \mathrm{mg} / \mathrm{kg}$ bw/day. Group IV (AINPs + SML): received SML followed (after 2 hours) by AINPs, at the dose levels indicated for group II and III. The exposure levels of SML and AINPs were based on previous studies of Hemalatha et al. )2013) and Jo et al. (2016), respectively.

AINPs was daily dispersed in bi-distilled water (at $\left.4^{\circ} \mathrm{C}\right)$ using ultrasonic vibration $(100 \mathrm{~W}, 30 \mathrm{kHz})$ for 30 min before administration to ensure homogeneous distribution. The solution was subjected to sonication and vigorous vortexing as per requirement, to ensure uniform suspension. Sesamol was freshly prepared daily by dissolving the required amount of sesamol powder in a suitable amount of distilled water (Hemalatha et al. 2013).

\subsection{Sampling}

At the end of experiment, blood samples were collected from the retro-orbital plexus, and were allowed to sit for 30 min, then centrifuged at $3000 \mathrm{rpm}$ at $4^{\circ} \mathrm{C}$ for $15 \mathrm{~min}$. Sera were collected and stored at $-80^{\circ} \mathrm{C}$ to be used for interleukine analysis.

Rats from all experimental groups were euthanized by cervical dislocation under sodium pentobarbital anesthesia (60 $\mathrm{mg} / \mathrm{kg}$ ). Brains were dissected and assigned into three sets. The first set was homogenized, using Teflon homogenizer in cold PBS ( $\mathrm{pH} 7.4)$, and the obtained homogenates were centrifuged at $4^{\circ} \mathrm{C}$ for $20 \mathrm{~min}(14,000 \times \mathrm{g})$. Supernatants were obtained and stored at $-80^{\circ} \mathrm{C}$ to be used for investigation of MDA, 8-OHdG and antioxidants. The second brain set was immediately frozen in liquid nitrogen and stored at $-80^{\circ} \mathrm{C}$ until being processed for RT-qPCR expression and assessment of AChE activity. The third set of brains was fixed in neutral buffered formalin $10 \%$ solution to be used in the histopathological investigation.

\subsection{Acetylcholinesterase (AChE)}

The brain acetylcholinesterase (AChE) activity was measured using ELISA Kit obtained from MyBioSource Biotechnology company, Egypt (Catalog No. MBS2501434), according to manufacturer instructions.

\subsection{Oxidative stress/antioxidant biomarkers}

The concentrations of the lipid peroxidation product malondialdehyde (MDA) and reduced glutathione (GSH), and the activities of catalase (CAT) and superoxide dismutase (SOD) were assessed in brain tissue using assay kits from the Biodiagnostic Company, Egypt, (Catalog No: MD2529, GR2511, CA2517, SD2521, respectively) following the manufacturer's instructions.

\subsection{8-hydroxy-2'-deoxyguanosine (8-OHdG)}


Brain 8-OHdG was quantified using 8-OHdG ELISA kit (Catalog No: MBS008851) obtained from MyBioSource Biotechnology company, Egypt.

\subsection{Inflammatory response markers}

Serum IL-1 $\beta$ and IL-6 levels were assessed using the commercial rat enzyme -linked immunosorbent assay (ELISA) kits obtained from MyBioSource, San Diego, California, USA (Catalog No: MBS825017 and MBS175908, respectively. The assessment protocols were followed as provided by the manufacturer.

\subsection{Quantitative real-time polymerase chain reaction (qRT-PCR) of Glutathione-S-transferase (GST), Tumor necrosis factor-alpha (TNF- a), and Caspase-3 genes}

Total RNA in brain samples was extracted using QIAmp RNA mini kit (Qiagen, Hilden, Germany) as indicated by the manufacturer. Total RNA purity and concentration were stermined using a nanodrop ND-2000 spectrophotometer. The isolated RNA was used for cDNA synthesis using reverse transcriptase (Fermentas, EU). Real-time PCR (qPCR) was performed in a total volume of $20-\mu$ l using a mixture of $1 \mu \mathrm{l}$ CDNA, $0.5 \mathrm{mM}$ of each primer (Table 1), iQ SYBR Green Premix (Bio-Rad 170-880, USA). PCR amplification and analysis were achieved using Bio-Rad iCycler thermal cycler and the MyiQ realtime PCR detection system. Each assay includes triplicate samples for each tested cDNAs and no-template negative control, the expression relative to control is calculated using the Eq. 2- $\Delta \Delta C T$ (Livak and Schmittgen, 2001).

Table 1

Primer sequences reference of GST, TNF-a and Caspase-3 genes of Rattus norvegicus

\begin{tabular}{|llll|}
\hline Target genes & Accession no. & Sequence (5' to 3') & Product size \\
\hline GAPDH & NM_017008.4 & F: 5'-GAGACAGCCGCATCTTCTTG-3' & 224bp \\
(reference gene) & & R: 5'-TGACTGTGCCGTTGAACTTG-3' & \\
\hline GST & NM_031509.2 & F:5'GACCAGAGCCATTCTCAACTACA3' & 261bp \\
& & R:5'CTCAGCCTATTGCCAACGAGATA3' & \\
\hline TNF-a & NM_012675.3 & F: 5'ACACACGAGACGCTGAAGTA3' & 235bp \\
& & R: 5'GGAACAGTCTGGGAAGCTCT3' & \\
\hline Casp-3 & NM_012922.2 & F: 5'-CATGCACATCCTCACTCGTG-3' & 158bp \\
& & R: 5'-CCCACTCCCAGTCATTCCTT-3' & \\
\hline
\end{tabular}

\subsection{Histopathological examination}

The brain of each rat was removed completely and fixed in $10 \%$ neutral buffered formalin solution for $72 \mathrm{~h}$. For histopathological investigation, samples were trimmed, washed, dehydrated, embedded in paraffin wax, serially sectioned with a microtome at $3 \mu \mathrm{m}$ thickness and stained with hematoxylin and eosin (H\&E) stain. Additionally, histological photos were taken using Lieca DMLB microscopes and Leica EC3 digital camera. The comparative histopathological lesion scoring in different brain regions was conducted according to Gibson-Corley et al. (2013).

\subsection{Data analysis}


The obtained data are presented as means \pm S.E of the mean. All experimental data were subjected to one-way analysis of variance (ANOVA) followed by Duncan's Multiple Range test for post hoc analysis using SPSS software, version 17 (IBM, USA). The level of significance was set at $P \leq 0.05$.

\section{Results}

\subsection{Characterization of AINPs}

As displayed in Fig. 1, the ultrasonicated aluminum oxide nanoparticles appeared as crystals, in the form of rods, with average length of $20-35 \mathrm{~nm}$, and average thickness of 3-6 nm.

\subsection{Brain acetylcholinesterase (AChE) activity}

We have observed that subacute daily oral administration of AINPs $(100 \mathrm{mg} / \mathrm{kg})$ for 28 days significantly $(p \leq 0.05)$ elevated the activity of brain AChE (Table 2). However, concomitant administration of SML (100 mg/kg) with AINPs reduced the elevated enzyme activity, compared to AINPs group, but significant difference from control value was still present.

Table 2

Effect of AINPs and/or SML on biochemical indices in brain of rats from all experimental groups.

\begin{tabular}{|lllll|}
\hline & Control & SML & AlNPs & AlNPs + Sesamol \\
\hline AChE $(\mathrm{ng} / \mathrm{ml})$ & $9.81 \pm 0.60^{\mathrm{c}}$ & $11.66 \pm 0.90^{\mathrm{bc}}$ & $18.01 \pm 0.94^{\mathrm{a}}$ & $13.17 \pm 0.82^{\mathrm{b}}$ \\
\hline $\mathrm{MDA}(\mathrm{nmol} / \mathrm{g})$ & $28.75 \pm 1.12^{\mathrm{c}}$ & $26.15 \pm 1.23^{\mathrm{c}}$ & $68.15 \pm 2.03^{\mathrm{a}}$ & $44.15 \pm 1.86^{\mathrm{b}}$ \\
\hline GSH (mg/g) & $87.75 \pm 2.73^{\mathrm{a}}$ & $84.74 \pm 2.5^{\mathrm{a}}$ & $45.59 \pm 1.65^{\mathrm{c}}$ & $76.22 \pm 2.03^{\mathrm{b}}$ \\
\hline CAT $(\mathrm{U} / \mathrm{g})$ & $2.04 \pm 0.086^{\mathrm{a}}$ & $2.06 \pm 0.053^{\mathrm{a}}$ & $1.53 \pm 0.070^{\mathrm{b}}$ & $1.89 \pm 0.048^{\mathrm{a}}$ \\
\hline SOD $(\mathrm{U} / \mathrm{g})$ & $21.56 \pm 0.99^{\mathrm{a}}$ & $20.94 \pm 1.01^{\mathrm{a}}$ & $10.48 \pm 0.75^{\mathrm{c}}$ & $13.18 \pm 0.82^{\mathrm{b}}$ \\
\hline $8 \mathrm{OHdG}$ & $8.33 \pm 0.39^{\mathrm{b}}$ & $8.25 \pm 0.41^{\mathrm{b}}$ & $14.33 \pm 0.62^{\mathrm{a}}$ & $8.93 \pm 0.27^{\mathrm{b}}$ \\
\hline
\end{tabular}

Values are means $\pm S E, n=5$. Means in the same row with different superscripts $(a, b$, and $c)$ are statistically significant ( $p$ 5 0.05). AINPs: Alumina nanoparticles, SML: Sesamol, AChE: Acetylcholinesterase, MDA: Malondialdehyde, GSH:

Glutathione, CAT: Catalase, SOD: Superoxide dismutase, 8-OHdG: 8-hydroxy-2' -deoxyguanosine.

\subsection{Oxidant/antioxidant biomarkers findings}

When compared to respective control values, the level of brain MDA level was significantly $(p \leq 0.05)$ elevated, while that of GSH was significantly reduced in AINPs treated rats. Additionally, the activities of CAT and SOD were significantly inhibited. The group of rats that received both AINPs and SML showed significant improvement in all the indices, in comparison to AINPs treated group. However, values were still significantly different from control group, except for CAT which showed complete recovery (Table 2).

Concerning brain GST gene expression, we observed significant 4.8-fold increase in the AINPs group, compared to control. In the combined treatment group (AINPs $+S M L$ ), the expression significantly decreased to $56.3 \%$ of the AINPs group, but the value was significantly different from control (Fig. 2A \& 2D).

\subsection{8-OHdG findings}


As shown in Table 2, AINPs-treated group presented significant elevation ( $\mathrm{p} \leq 0.05)$ of brain 8-OHdG concentration, relative to control. However, concurrent administration of SML with AINPs completely recovered the level of 8-OHdG to control value.

\subsection{Inflammatory response findings}

As summarized in Table 3, IL-1 $\beta$ and IL-6 levels in serum of AINPs-intoxicated rats were significantly $(p \leq 0.05)$ elevated, with respect to corresponding controls. When SML was cosupplemented with AINPs, it alleviated the effect on both interleukines, compared to AINPs-treated group. However, the values were still higher than corresponding controls.

In addition, we recorded 3.9-fold increase in the expression of TNF-a gene in the brain of AINPs-treated animals, in comparison to control. However, the concomitant administration of SML with AINPs attenuated the expression to be $58.97 \%$ of the AINPs value, although still significantly higher than control value (Fig. 2B \& 2E).

Table 3

Effect of AINPs and/or SML on serum proinflammatory cytokines.

\begin{tabular}{|lcccc|}
\hline & Control & SML & AlNPs & AINPs + SML \\
\hline $\mathrm{IL}-1 \beta(\mathrm{pg} / \mathrm{ml})$ & $200.40 \pm 3.70^{\mathrm{c}}$ & $194.60 \pm 3.23^{\mathrm{c}}$ & $235.00 \pm 4.01^{\mathrm{a}}$ & $211.60 \pm 3.78^{\mathrm{b}}$ \\
\hline $\mathrm{IL}-6(\mathrm{pg} / \mathrm{ml})$ & $314.00 \pm 3.63^{\mathrm{c}}$ & $318.00 \pm 3.33^{\mathrm{c}}$ & $436.20 \pm 5.14^{\mathrm{a}}$ & $368.80 \pm 4.19^{\mathrm{b}}$ \\
\hline $\begin{array}{l}\text { Values are means } \pm \text { SE, } \mathrm{n}=5 \text {. Means in the same row with different superscripts }(\mathrm{a}, \mathrm{b}, \text { and } \mathrm{c}) \text { are statistically significant } \\
(\mathrm{p} \leq 0.05) \text {. AlNPs: Alumina nanoparticles, SML: Sesamol. }\end{array}$ & & \\
\hline
\end{tabular}

\subsection{Brain caspase-3 finding}

As indicated in (Fig. 2C \& F), caspase-3 gene expression presented 4.4-fold increase in the brain of AlNPs-treated animals, relative to control. However, the concurrent administration of SML with AINPs ameliorated this overexpression to be $52.27 \%$ of the AINPs group, although still significantly higher than control.

\subsection{Histopathological findings}

Histopathologically, all examined brain regions in negative control group and in SML treated group (Fig. 3) showed a normal histological architecture. On the other hand, AINPs administration induced necrosis and complete loss of several Purkinje cells; congestion and edema in molecular layer of the cerebellum (Fig. 4a). Medulla oblongata showed multiple hemorrhage, edema, neuronal necrosis, microglia activation (Fig. 4c) and apoptosis on neurons (Fig. 4e). In cerebrum, AINPs toxicity induced a widespread perivascular edema, hyalinization of vascular walls, a widespread apoptosis of neurons, neuronal necrosis, and edema in neuropil (Fig. 5a \& 5c). Additionally, midbrain red nucleus showed a neuronal degeneration of multiple neurons (Fig. 5e).

As summarized in Table 4, cosupplementation of SML with AINPs alleviated the histopathological alterations induced by the nanometal. The brain tissues showed necrosis of a few Purkinje cells in cerebellum (Fig. 4b). In medulla oblongata, mild neuronal necrosis and edema were observed (Fig. 4d \& 4f). In cerebrum, a slight perivascular edema and a few apoptotic neurons were detected (Fig. 5b). In midbrain red nucleus, neuronal degeneration of a few neurons was recorded (Fig. 5f). 
Table 4

A comparative lesion scoring in examined brain regions between different groups.

\begin{tabular}{|c|c|c|c|c|c|c|c|c|c|c|c|c|c|c|c|c|}
\hline \multirow{2}{*}{$\begin{array}{l}\text { Brain regions } \\
\text { Lesions }\end{array}$} & \multicolumn{4}{|c|}{ Cerebellum } & \multicolumn{4}{|c|}{ Medulla oblongata } & \multicolumn{4}{|c|}{ Cerebrum } & \multicolumn{4}{|c|}{ Midbrain red nucleus } \\
\hline & G1 & G2 & G3 & G4 & G1 & G2 & G3 & G4 & G1 & G2 & G3 & G4 & G1 & G2 & G3 & G4 \\
\hline Hemorrhage & - & - & - & - & - & - & +++ & - & - & - & - & - & - & - & - & - \\
\hline Degeneration & - & - & +++ & + & - & - & +++ & ++ & - & - & ++ & + & - & - & +++ & + \\
\hline Necrosis & - & - & +++ & + & - & - & ++ & + & - & - & ++ & - & - & - & - & - \\
\hline Apoptosis & - & - & - & - & - & - & + & - & - & - & ++++ & + & - & - & - & - \\
\hline Edema & - & - & + & - & - & - & ++ & + & - & - & ++ & + & - & - & - & - \\
\hline
\end{tabular}

G1, G2, G3, and G4 represent negative control group, SML treated group, AINPs treated group, and SML + AINPs treated groups, respectively.

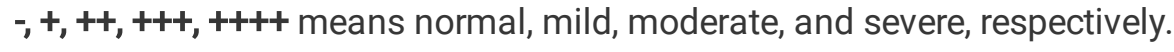

\section{Discussion}

Excessive or long-term exposure to aluminum nanoparticles (AINPs) constitutes a major hazard to human and animal nervous system. Thus, search is continuous for phytochemicals with neuroprotective effects. In the present study, we have studied the impact of sesamol (SML) co-supplementation on the AINPs induced toxic effects in brain of rats. The small size of AINPs and their large surface area enable them to penetrate biological membranes easily through all routes (De et al. 2020). In the current investigation, we adopted the oral route of administration, as it is the most common route of exposure. Our results regarding TEM photomicrographs revealed that the AINPs were rods with average length of 20-35 $\mathrm{nm}$ and average thickness of 3-6 $\mathrm{nm}$, which were approximately within the range of $<50 \mathrm{~nm}$, as provided by the manufacturer.

Acetylcholinesterase (AChE) ia a key enzyme involved in catalyzing acetylcholine into choline and acetic acids at nerve synapses and neuromuscular junctions. Our findings demonstrated that oral administration of AINPs to rats for 28 days increased the activity of brain AChE, which is expected to reduce the level of brain acetylcholine. This may contribute to AINPs-induced neurodegeneration, as the onset of degenerative diseases such as Alzheimer's disease was linked to reduced acetylcholine level (Emmett and Greenfield, 2004). The elevated AChE activity may result from allosteric interaction of $\mathrm{Al}^{3+}$ cation with the enzyme peripheral sites causing modifications in the enzyme secondary structure, and thus enhances its activity (Zatta et al. 1994). Our findings are in accordance with previous studies demonstrating elevated brain AChE activity in response to aluminum treatment (Auti and Kulkarni 2019; Aboelwafa et al. 2020; Liu et al. 2020b).

Of note, when SML was coadministered with AINPs, it lowered the elevated AChE activity, reflecting the neuroprotective effect of SML. Likewise, SML was previously reported to produce similar effect in experimental paradigm of diabetes associated cognitive decline (Kuhad and Chopra 2008) and Alzheimer's disease induced by intracerebroventricular injection of streptozotocin (ICV-STZ) in rats (Sachdeva et al. 2015).

The brain is more susceptible to oxidative stress due to poor antioxidant content, high oxidizable polyunsaturated fatty acids and iron content, and great metabolic rate (Casetta et al. 2005). Herein, we recorded elevation of brain MDA, depletion of GSH, and inhibition of activities of SOD and CAT in AINPs treated rats. Consistent with our results, Manke et al. (2013) showed that ROS generation with consequent oxidative stress is the major mechanism of nanoparticles-induced toxicity, due to large surface area, compared to their larger counterparts. Similar to our results, AINPs administered orally to rats was shown to increase lipid peroxidation and deplete GSH with suppression of GPx and SOD activities in brain 
(Arslanbaş and Coşar 2019; De et al. 2020; Zhang et al. 2021a). In addition, brain oxidative stress was reported in rats received AINPs intraperitoneal (Li et al. 2009; Morsy et al. 2016), intravenous (Mrad et al. 2017), or via intranasal instillation (Ji et al. 2011).

The elevated level of MDA with depletion of GSH indicated redox imbalance. MDA, a lipid peroxidation marker, is a toxic adduct which contributes to neuronal cell death (Serteser et al. 2002). Low GSH level could be attributed to excessive utilization in scavenging ROS (De et al. 2020). The reduced activity of CAT and SOD may result from being utilized in detoxication of $\mathrm{H}_{2} \mathrm{O}_{2}$ and superoxide radical (Abou-Zeid et al. 2021). We also observed upregulation of brain GST gene expression in AINPs intoxicated rats. This may occur in response to increased ROS generation. The increased utilization of GSH by GST enzyme, may contribute to the recorded GSH depletion. In agreement with our findings, Prabhakar et al. (2012) reported increased GST in brain of AINPs-treated rats, while GSH concentration was decreased.

Interestingly, when AINPs - treated rats were co-supplemented with SML, amelioration of all biochemical indices of oxidative stress was observed, reflecting the antioxidant potential of SML. This could be attributed to the capacity of SML to scavenge hydroxyl, superoxide, NO, ABTS and DPPH radicals (Kanimozhi and Prasad 2009; Mishra et al. 2011) through its phenolic hydroxyl group (Majdalawieh and Mansour 2019). SML exhibited 1.8-fold greater radical scavenging than ascorbic acid (Majdalawieh and Mansour, 2019) and 20 times stronger antioxidant ability compared to melatonin (Mishra et al. 2011).

In agreement with our findings, SML reduced lipid peroxidation and improved GSH and enzymatic antioxidants in brain of rat experimental paradigm of diabetes (VanGilder et al. 2009), Alzheimer's disease (Sachdeva et al. 2015), Huntington's disease (Kumar et al 2010), chronic intermittent hypoxia (Zhang et al. 2021b), and focal cerebral ischemia/reperfusion injury (Gao et al. 2017). The induction of antioxidant enzymes by sesamol was thought to be mediated through activation of Nrf2 transcriptional pathway and its nuclear translocation (Ren et al. 2018) and activation of SIRT1-SIRT3-FOXO3a expression (Ruankham et al. 2021).

Moreover, we demonstrated elevation of brain 8-OHdG concentration in AINPs intoxicated animals, reflecting oxidative DNA damage. In accordance with our results, previous studies documented the ability of AINPs to induce DNA damage in mouse brain (De et al. 2020) bone marrow and sperm (Zhang et al. 2017) secondary to oxidative stress. Notably, when SML was coadministered with AINPs, it reduced the brain 8-OHdG level. Similar to our findings, SML was reported to inhibit the radiation-induced DNA damage both in vivo and in vitro (Prasad et al. 2005; Kumar et al. 2018).

Accumulation of ROS may initiate apoptosis (Hsin et al. 2008). Our findings showed upregulation of brain caspase-3 in brain of rats intoxicated with AINPs. Similar to our results, Nano- $\mathrm{Al}_{2} \mathrm{O}_{3}$ produced apoptosis manifested by downregulation of bcl-2 and upregulation of p53 and Bax gene expression in brain of rats (Liu et al. 2020a), and by caspase-3 gene overexpression in mouse brain (Zhang et al., 2011). Similar effect was demonstrated in vitro in astrocytes of neonatal rat cerebral cortex (Dong et al. 2019) and mouse neuroblastoma cells (Nogueira et al. 2019). The AINPs induced apoptosis seems to be mediated via mitochondrial (intrinsic) pathway via activation of p53 pathway consequent to increased oxidative DNA damage (Liu et al. 2020b). Our histopathological findings revealed a wide spread neuronal apoptosis in the cerebral neurons in the AINPs treated group, which is in line with the upregulation of caspase-3 gene.

Of note, coadministration of SML with AINPs downregulated the expression of caspase-3 in brain. Similar effect of SML was reported in brain of rats with rotenone-induced Parkinson's disease (Sonia Angeline et al. 2013), focal cerebral ischemia/reperfusion injury (Gao et al. 2017), and experimental diabetes (Chopra et al. 2010). The molecular mechanisms underlying the antiapoptotic activity of SML in nerve cells may include activating SIRT1-SIRT3-FOX03a expression, upregulating of the anti-apoptotic protein Bcl-2 and inhibition of the proapoptotic protein Bax (Ruankham et al. 2021).

It has been documented that oxidative damage is a major initiator of inflammatory cascade via several mechanisms (Manke et al. 2013). We recorded overexpression of brain TNF- $a$ gene and increased levels of IL1- $\beta$ and IL-6 in serum of 
AINPs-intoxicated rats, reflecting inflammatory response which may contribute to development of neurodegenerative deficits (Win-Shwe and Fujimaki 2011; Guo et al. 2018). Similar to our results, AINP increased the levels of proinflammatory cytokines such as IL-1ß, IL-6 and TNF-a in brain of rats (Arslanbaş and Coşar 2019; Liu et al. 2020a; Zhang et al. 2021a) and in vitro in astrocytes of neonatal rats (Dong et al. 2019). Compelling evidence has suggested activation of NF-KB as a major pathway of aluminum induced neuroinflammation (Zhang et al. 2018). Yun et al. (2020) have shown that the activation of notch pathway is the main mechanism of lung inflammation induced by AINPs.

Importantly, concomitant SML treatment with AINPs alleviated the inflammatory response as evidenced by downregulation of brain TNF-a expression and reduction of IL-1 $\beta$ and IL- 6 levels. This is in line with the previous studies reporting the anti-inflammatory activity of SML in brain of rats with diabetic neuropathy (Chopra et al., 2010; Misra et al. 2011; Sachdeva et al. 2015), focal cerebral ischemia/reperfusion injury (Gao et al. 2017), and chronic intermittent hypoxia (Zhang et al. 2021b), and in lungs of rats with endotoxemia (Chu et al. 2010). The anti-inflammatory effect of SML was suggested to be mediated via suppressing NF-KB/MAPK activation and upregulating AMP kinase signaling (Wu et al. 2015; Liu et al. 2017).

Histopathologically, a notable neuronal degeneration and/ or necrosis was observed in different areas in the brain of AINPs treated group. Interestingly, the most affected neurons were motor neurons (motor neurons of medulla oblongata and midbrain red nucleus) or neurons with an inhibitory action on certain motor neurons (Purkinje cells of cerebellum). Hemorrhages and inflammatory edema were recorded especially in medulla oblongata. Additionally, perivascular edema and hyalinization of vascular walls was observed in the cerebellum. The nanosized aluminum can easily penetrate the blood brain barrier and accumulate in brain tissues (De et al. 2020) resulting generation of ROS. The latter may produce oxidative damage on lipids in cell and mitochondrial membranes and on cellular macromolecules like DNA and protein, and thus induce histopathological changes with degradation of neurons. Our findings are in agreement with previous reports demonstrating histopathological changes in brain of rats and mice (Morsy et al. 2016; Mrad et al. 2017; De et al. 2020; Liu et al. 2020; Zhang e tal. 2021a). Finally, all the observed histopathological alterations in brain tissue of AINPs treated rats have been ameliorated upon concomitant administration of SML with AINPs. This protective effect of SML, is in line with our findings concerning the oxidative stress markers and inflammatory cytokines.

\section{Conclusions}

In conclusion, administration of AINPs to rats at $100 \mathrm{mg} / \mathrm{kg}$ was associated with neurotoxicity and brain damage. We hypothesized oxidative damage, apoptosis and inflammatory response as underling mechanisms. Concomitant administration of SML with AINPs exerted neuroprotective effect probably through the antioxidant, anti-apoptotic and antiinflammatory activities of SML. The present study presents SML as a protective agent from AINPs-induced neurodegenerative disorders.

\section{Declarations}

\section{Ethics approval and consent to participate}

Ethics approval and consent to participate this study was approved by the Institutional Animal Care and Use Committee (IACUC) (Approval number: VUSC-007-1-20), Faculty of Veterinary Medicine, University of Sadat City, Egypt.

\section{Consent for publication:}

All the authors have given the consent to publish the paper. 


\section{Availability of data and materials:}

Not applicable.

\section{Competing interests:}

The authors declare that there are no conflicts of interest.

\section{Funding:}

The authors declare that there are no sources of funding to be acknowledged.

\section{Authors' contributions}

Shimaa Abou-Zeid: conceptualization, investigation, data analysis, writing original draft. Basma Elkhadrawey: methodology. Anis Anis: histopathological examination, writing original draft. Huda AbuBakr: gene expression study, writing original draft. Badr El-Bialy: methodology and revising the manuscript. Hesham Elsabbagh: conceived the idea and managed the study. Nermeen El-Borai: conceptualization, investigation, data analysis, writing original draft. All authors have contributed to writing this article, editing, and have critically read and revised the manuscript, and approved its submission for publication.

\section{Acknowledgements}

We thank consult of Central Labs in both Faculty of Veterinary Medicine, University of Sadat City \& Faculty of Veterinary Medicine, Cairo University, as we had bench space to work.

\section{References}

1. Aboelwafa HR, El-Kott AF, Abd-Ella EM, Yousef HN (2020) The possible neuroprotective effect of silymarin against aluminum chloride-prompted Alzheimer's-like disease in rats. Brain Sci 10:628. https://doi.org/10.3390/brainsci10090628

2. Abou-Zeid SM, Tahoun EA, AbuBakr HO (2021) Ameliorative effects of jojoba oil on fipronil-induced hepatorenal- and neuro-toxicity: the antioxidant status and apoptotic markers expression in rats. Environ Sci Pollut Res Int. https://doi.org/10.1007/s11356-020-12083-2

3. Abou-Zeid SM, AbuBakr HO, Mohamed MA, El-Bahrawy A (2018) Ameliorative effect of pumpkin seed oil against emamectin induced toxicity in mice. Biomed Pharmacother 98:242-251.

https://doi.org/10.1016/j.biopha.2017.12.040

4. Auti ST, Kulkarni YA (2019) Neuroprotective effect of cardamom oil against aluminum induced neurotoxicity in rats. Front Neurol 10:399. https://doi.org/10.3389/fneur.2019.00399

5. Casetta I, Govoni V, Granieri E (2005) Oxidative stress, antioxidants and neurodegenerative diseases. Curr Pharm Des 11:2033-2052. https://doi.org/10.2174/1381612054065729

6. Chen L, Yokel RA, Hennig B, Toborek M (2008) Manufactured aluminum oxide nanoparticles decrease expression of tight junction proteins in brain vasculature. J Neuroimmune Pharmacol 4:286-295. https://doi.org/10.1007/s11481008-9131-5 
7. Chopra K, Tiwari V, Arora V, Kuhad A (2010) Sesamol suppresses neuro-inflammatory cascade in experimental model of diabetic neuropathy. J Pain 10:950-957. https://doi.org/10.1016/j.jpain.2010.01.006

8. Chu PY, Chien SP, Hsu DZ, Liu MY (2010) Protective effect of sesamol on the pulmonary inflammatory response and lung injury in endotoxemic rats. Food Chem Toxicol 48:1821-1826. https://doi.org/10.1016/j.fct.2010.04.014

9. Arslanbaş E, COŞAR Z (2019) Toxic effects of cutaneous and oral exposure to aluminum and magnesium nanoparticles on brain tissue in rats. Ankara Üniversitesi Veteriner Fakültesi Dergisi.

https://doi.org/10.33988/auvfd.569990

10. De A, Ghosh S, Chakrabarti M, Ghosh I, Banerjee R, Mukherjee A (2020) Effect of low-dose exposure of aluminium oxide nanoparticles in Swiss albino mice: Histopathological changes and oxidative damage. Toxicol Ind Health 36:567-579. https://doi.org/10.1177/0748233720936828

11. Dey S, Bakthavatchalu V, Tseng MT, Wu P, Florence RL, Grulke EA, Yokel RA, Dhar SK, Yang HS, Chen Y, St Clair DK (2008) Interactions between SIRT1 and AP-1 reveal a mechanistic insight into the growth promoting properties of alumina (Al203) nanoparticles in mouse skin epithelial cells. Carcinogenesis 29:1920-1929.

https://doi.org/10.1093/carcin/bgn175

12. Dong L, Tang S, Deng F, Gong Y, Zhao K, Zhou J, Liang D, Fang J, Hecker M, Giesy JP, Bai X, Zhang H (2019) Shapedependent toxicity of alumina nanoparticles in rat astrocytes. Sci Total Environ 690:158-166. https://doi.org/10.1016/j.scitotenv.2019.06.532

13. Emmett SR, Greenfield SA (2004) A peptide derived from the C-terminal region of acetylcholinesterase modulates extracellular concentrations of acetylcholinesterase in the rat substantia nigra. Neurosci Lett 358:210-214. https://doi.org/10.1016/j.neulet.2003.12.078

14. Farag MR, Mahmoud HK, El-Sayed SAA, Ahmed SYA, Alagawany M, Abou-Zeid SM (2021) Neurobehavioral, physiological and inflammatory impairments in response to bifenthrin intoxication in Oreochromis niloticus fish: Role of dietary supplementation with Petroselinum crispum essential oil. Aquat Toxicol 231:105715.

https://doi.org/10.1016/j.aquatox.2020.105715

15. Gao XJ, Xie GN, Liu L, Fu ZJ, Zhang ZW, Teng LZ (2017) Sesamol attenuates oxidative stress, apoptosis and inflammation in focal cerebral ischemia/reperfusion injury. Exp Ther Med 14:841-847. https://doi.org/10.3892/etm.2017.4550

16. Gibson-Corley KN, Olivier AK, Meyerholz DK (2013) Principles for valid histopathologic scoring in research. Vet Pathol 50:1007-1015

17. Guo JD, Zhao X, Li Y, Li GR, Liu XL (2018) Damage to dopaminergic neurons by oxidative stress in Parkinson's disease (Review). Int J Mol Med 41:1817-1825. https://doi.org/10.3892/ijmm.2018.3406

18. Hassanzadeh P, Arbabi E, Rostami F (2014) The ameliorative effects of sesamol against seizures, cognitive impairment and oxidative stress in the experimental model of epilepsy. Iran J Basic Med Sci 17:100-107

19. Hemalatha G, Pugalendi KV, Saravanan R (2013) Modulatory effect of sesamol on DOCA-salt-induced oxidative stress in uninephrectomized hypertensive rats. Mol Cell Biochem 379:255-265. https://doi.org/10.1007/s11010-013-1647-1

20. Hsin YH, Chen CF, Huang S, Shih TS, Lai PS, Chueh PJ (2008) The apoptotic effect of nanosilver is mediated by a ROS- and JNK-dependent mechanism involving the mitochondrial pathway in NIH3T3 cells. Toxicol Lett 179:130139. https://doi.org/10.1016/j.toxlet.2008.04.015

21. Jan KC, Ho CT, Hwang LS (2008) Bioavailability and tissue distribution of sesamol in rat. J Agric Food Chem 56:7032-7037. https://doi.org/10.1021/jf8012647

22. Ji JW, Zhang QL, Bai R, Gao FP, Ge CC, Wang ZW, Chen CY, Zhang C, Niu Q (2011) Changes of brain oxidative stress induced by nano-alumina in ICR mice. Zhonghua Lao Dong Wei Sheng Zhi Ye Bing Za Zhi 29:434-436

23. Jo E, Seo GB, Kim H, Choi K, Kwon JT, Kim P, Eom I (2016) Toxic effects of alumina nanoparticles in rat cerebrums and kidneys. Journal of Environmental Health Sciences 42:27-33. https://doi.org/10.5668/JEHS.2016.42.1.27

Page 12/18 
24. Kanimozhi P, Prasad NR (2009) Antioxidant potential of sesamol and its role on radiation-induced DNA damage in whole-body irradiated Swiss albino mice. Environ Toxicol Pharmacol 28:192-197.

https://doi.org/10.1016/j.etap.2009.04.003

25. Krause B, Kriegel F, Rosenkranz D, Dreiack N, Tentschert J, Jungnickel H, Jalili P, Fessard V, Laux P, Luch A (2020) Aluminum and aluminum oxide nanomaterials uptake after oral exposure - a comparative study. Sci Rep 10, 2698 (2020). https://doi.org/10.1038/s41598-020-59710-z

26. Kuhad A, Chopra K (2008) Effect of sesamol on diabetes-associated cognitive decline in rats. Exp Brain Res 185:411 - 20. https://doi.org/10.1007/s00221-007-1166-y

27. Kumar A, Choudhary S, Adhikari JS, Chaudhury NK (2018) Sesamol ameliorates radiation induced DNA damage in hematopoietic system of whole body $\mathrm{Y}$-irradiated mice. Environ Mol Mutagen 59:79-90.

https://doi.org/10.1002/em.22118

28. Kumar P, Kalonia H, Kumar A (2010) Protective effect of sesamol against 3-nitropropionic acid-induced cognitive dysfunction and altered glutathione redox balance in rats. Basic Clin Pharmacol Toxicol 107:577-582. https://doi.org/10.1111/j.1742-7843.2010.00537.x

29. Li H, Huang T, Wang Y, Pan B, Zhang L, Zhang Q, Niu Q (2020) Toxicity of alumina nanoparticles in the immune system of mice. Nanomedicine (Lond) 15:927-946. https://doi.org/10.2217/nnm-2020-0009

30. Li XB, Zheng H, Zhang ZR, Li M, Huang ZY, Schluesener HJ, Li YY, Xu SQ (2009) Glia activation induced by peripheral administration of aluminum oxide nanoparticles in rat brains. Nanomedicine 5:473-479.

https://doi.org/10.1016/j.nano.2009.01.013

31. Liu L, Liu Y, Zhao J, Xing X, Zhang C, Meng H (2020b) Neuroprotective Effects of D-(-)-Quinic Acid on Aluminum Chloride-Induced Dementia in Rats. Evid Based Complement Alternat Med. May 11;2020:5602597. https://doi.org/10.1155/2020/5602597

32. Liu Z, Chen Y, Qiao Q, Sun Y, Liu Q, Ren B, Liu X (2017) Sesamol supplementation prevents systemic inflammationinduced memory impairment and amyloidogenesis via inhibition of nuclear factor kappaB. Mol Nutr Food Res 61:5,1600734. https://doi.org/10.1002/mnfr.201600734

33. Liu H, Zhang W, Fang Y, Yang H, Tian L, Li K, Lai W, Bian L, Lin B, Liu X, Xi Z (2020a) Neurotoxicity of aluminum oxide nanoparticles and their mechanistic role in dopaminergic neuron injury involving p53-related pathways. $J$ Hazard Mater 392:2020a,122312. https://doi.org/10.1016/j.jhazmat.2020.122312

34. Livak KJ, Schmittgen HD (2001) Analysis of relative gene expression data using realtime quantitative PCR and the 2(Delta Delta C(T)) method. Methods 25:402-408. https://doi.org/10.1006/meth.2001.1262

35. Majdalawieh AF, Mansour ZR (2019) Sesamol, a major lignan in sesame seeds (Sesamum indicum): Anti-cancer properties and mechanisms of action. Eur J Pharmacol 855:75-89. https://doi.org/10.1016/j.ejphar.2019.05.008

36. Manke A, Wang L, Rojanasakul Y (2013) Mechanisms of nanoparticle-induced oxidative stress and toxicity. Biomed Res Int 2013:942916. https://doi.org/10.1155/2013/942916

37. Mishra K, Srivastava PS, Chaudhury NK (2011) Sesamol as a potential radioprotective agent: in vitro studies. Radiat Res 176:613-623. https://doi.org/10.1667/rr2661.1

38. Misra S, Tiwari V, Kuhad A, Chopra K (2011) Modulation of nitrergic pathway by sesamol prevents cognitive deficits and associated biochemical alterations in intracerebroventricular streptozotocin administered rats. Eur J Pharmacol 659:177-186. https://doi.org/10.1016/j.ejphar.2011.03.026

39. Morsy GM, Abou El-Ala KS, Ali AA (2016) Studies on fate and toxicity of nanoalumina in male albino rats: Oxidative stress in the brain, liver and kidney. Toxicol Ind Health 32:200-214. https://doi.org/10.1177/0748233713498462

40. Mrad I, Sakly M, Amara S (2017) Aluminum Oxide Nanoparticles Induced Cognitive Deficits and Oxidative Stress in Frontal Cortex and Cerebellum of Rat. Adv J Toxicol Curr Res 1:007-014

Page $13 / 18$ 
41. Nogueira D, Arl M, Köerich J, Zitta C, Ouriques L, Vicentini D, Matias W (2019) Comparison of cytotoxicity of a-Al203 and $\eta$-Al2O3 nanoparticles toward neuronal and bronchial cells. Toxicol in Vitro 61:104596.

https://doi.org/10.1016/j.tiv.2019.104596

42. Prabhakar PV, Reddy UA, Singh SP, Balasubramanyam A, Rahman MF, Indu Kumari S, Agawane SB, Murty US, Grover P, Mahboob M (2012) Oxidative stress induced by aluminum oxide nanomaterials after acute oral treatment in Wistar rats. J Appl Toxicol 32:436-445. https://doi.org/10.1002/jat.1775

43. Prasad NR, Menon VP, Vasudev V, Pugalendi KV (2005) Radioprotective effect of sesamol on gamma-radiation induced DNA damage, lipid peroxidation and antioxidants levels in cultured human lymphocytes. Toxicology 209:225-235. https://doi.org/10.1016/j.tox.2004.12.009

44. Ren B, Yuan T, Zhang X, Wang L, Pan J, Liu Y, Zhao B, Zhao W, Liu Z, Liu X (2020) Protective Effects of Sesamol on Systemic Inflammation and Cognitive Impairment in Aging Mice. J Agric Food Chem 68:3099-3111. https://doi.org/10.1021/acs.jafc.9b07598

45. Ren B, Yuan T, Diao Z, Zhang C, Liu Z, Xuebo L (2018) Protective effects of sesamol on systemic oxidative stressinduced cognitive impairments via regulating Nrf2/Keap1 pathway. Food Function. 9.10.1039/C8F001436A

46. Ruankham W, Suwanjang W, Wongchitrat P, Prachayasittikul V, Prachayasittikul S, Phopin K (2021) Sesamin and sesamol attenuate $\mathrm{H} 2 \mathrm{O} 2$-induced oxidative stress on human neuronal cells via the SIRT1-SIRT3-FOXO3a signaling pathway. Nutr Neurosci 24:90-101. https://doi.org/10.1080/1028415X.2019.1596613

47. Sachdeva AK, Misra S, Pal Kaur I, Chopra K (2015) Neuroprotective potential of sesamol and its loaded solid lipid nanoparticles in ICV-STZ-induced cognitive deficits: behavioral and biochemical evidence. Eur J Pharmacol 15:747:132 - 40. https://doi.org/10.1016/j.ejphar.2014.11.014

48. Sengul AB, Asmatulu E (2020) Toxicity of metal and metal oxide nanoparticles: a review. Environ Chem Lett 18:16591683. https://doi.org/10.1007/s10311-020-01033-6

49. Serteser M, Ozben T, Gumuslu S, Balkan S, Balkan E (2002) Lipid peroxidation in rat brain during focal cerebral ischemia: prevention of malondialdehyde and lipid conjugated diene production by a novel antiepileptic, lamotrigine. Neurotoxicol 23:111-119. https://doi.org/10.1016/s0161-813x(02)00018-9

50. Shah SA, Yoon GH, Ahmad A, Ullah F, Ul Amin F, Kim MO (2015) Nanoscale-alumina induces oxidative stress and accelerates amyloid beta $(A \beta)$ production in ICR female mice. Nanoscale 7:15225-15237. https://doi.org/10.1039/c5nr03598h

51. Sonia Angeline M, Sarkar A, Anand K, Ambasta RK, Kumar P (2013) Sesamol and naringenin reverse the effect of rotenone-induced PD rat model. Neuroscience 254:379-394. https://doi.org/10.1016/j.neuroscience.2013.09.029

52. VanGilder RL, Kelly KA, Chua MD, Ptachcinski RL, Huber JD (2009) Administration of sesamol improved blood-brain barrier function in streptozotocin-induced diabetic rats. Exp Brain Res 197:23-34. https://doi.org/10.1007/s00221009-1866-6

53. Whyte KA, Greenfield SA (2003) Effects of acetylcholinesterase and butyrylcholinesterase on cell survival, neurite outgrowth, and voltage-dependent calcium currents of embryonic ventral mesencephalic neurons. Exp Neurol 184:496-509. https://doi.org/10.1016/s0014-4886(03)00386-8

54. Win-Shwe TT, Fujimaki H (20111) Nanoparticles and neurotoxicity. Int J Mol Sci 12:6267-80. https://doi.org/10.3390/ijms12096267

55. Wu XL, Liou CJ, Li ZY, Lai XY, Fang LW, Huang WC (2015) Sesamol suppresses the inflammatory response by inhibiting NF-KB/MAPK activation and upregulating AMP kinase signaling in RAW 264.7 macrophages. Inflamm Res 64:577-588. https://doi.org/10.1007/s00011-015-0836-7

56. Xiong S, Mu T, Wang G, Jiang X (2014) Mitochondria-mediated apoptosis in mammals. Protein Cell 5:737 - 49. https://doi.org/10.1007/s13238-014-0089-1 
57. Yun J, Yang H, Li X, Sun H, Xu J, Meng Q, Wu S, Zhang X, Yang X, Li B, Chen R (2020) Up-regulation of miR-297 mediates aluminum oxide nanoparticle-induced lung inflammation through activation of Notch pathway. Environ Pollut 259:113839. https://doi.org/10.1016/j.envpol.2019.113839

58. Zatta P, Zambenedetti P, Bruna V, Filippi B (1994) Activation of acetylcholinesterase by aluminium(III): the relevance of the metal species. Neuroreport 5:1777-1780. https://doi.org/10.1097/00001756-199409080-00023

59. Zhang H, Jiao W, Cui H, Sun Q, Fan H (2021a) Combined exposure of alumina nanoparticles and chronic stress exacerbates hippocampal neuronal ferroptosis via activating IFN-y/ASK1/JNK signaling pathway in rats. J Hazard Mater 411:2021,125179. https://doi.org/10.1016/j.jhazmat.2021.125179

60. Zhang $P$, Wang $Y$, Wang $H$, Cao J (2021b) Sesamol alleviates chronic intermittent hypoxia-induced cognitive deficits via inhibiting oxidative stress and inflammation in rats. Neuroreport 32:105-111.

https://doi.org/10.1097/WNR.0000000000001564

61. Zhang Q, Wang H, Ge C, Duncan J, He K, Adeosun SO, Xi H, Peng H, Niu Q (2017) Alumina at 50 and 13 nm nanoparticle sizes have potential genotoxicity. J Appl Toxicol 37:1053-1064. https://doi.org/10.1002/jat.3456

62. Zhang H, Wang P, Yu H, Yu K, Cao Z, Xu F, Yang X, Song M, Li Y (2018) Aluminum trichloride-induced hippocampal inflammatory lesions are associated with IL-1 $\beta$-activated IL-1 signaling pathway in developing rats. Chemosphere 203:170-178. https://doi.org/10.1016/j.chemosphere.2018.03.162

63. Zhang QL, Li MQ, Ji JW, Gao FP, Bai R, Chen CY, Wang ZW, Zhang C, Niu Q (2011) In vivo toxicity of nano-alumina on mice neurobehavioral profiles and the potential mechanisms. Int J Immunopathol Pharmacol 24(1 Suppl):23S-29S

\section{Figures}
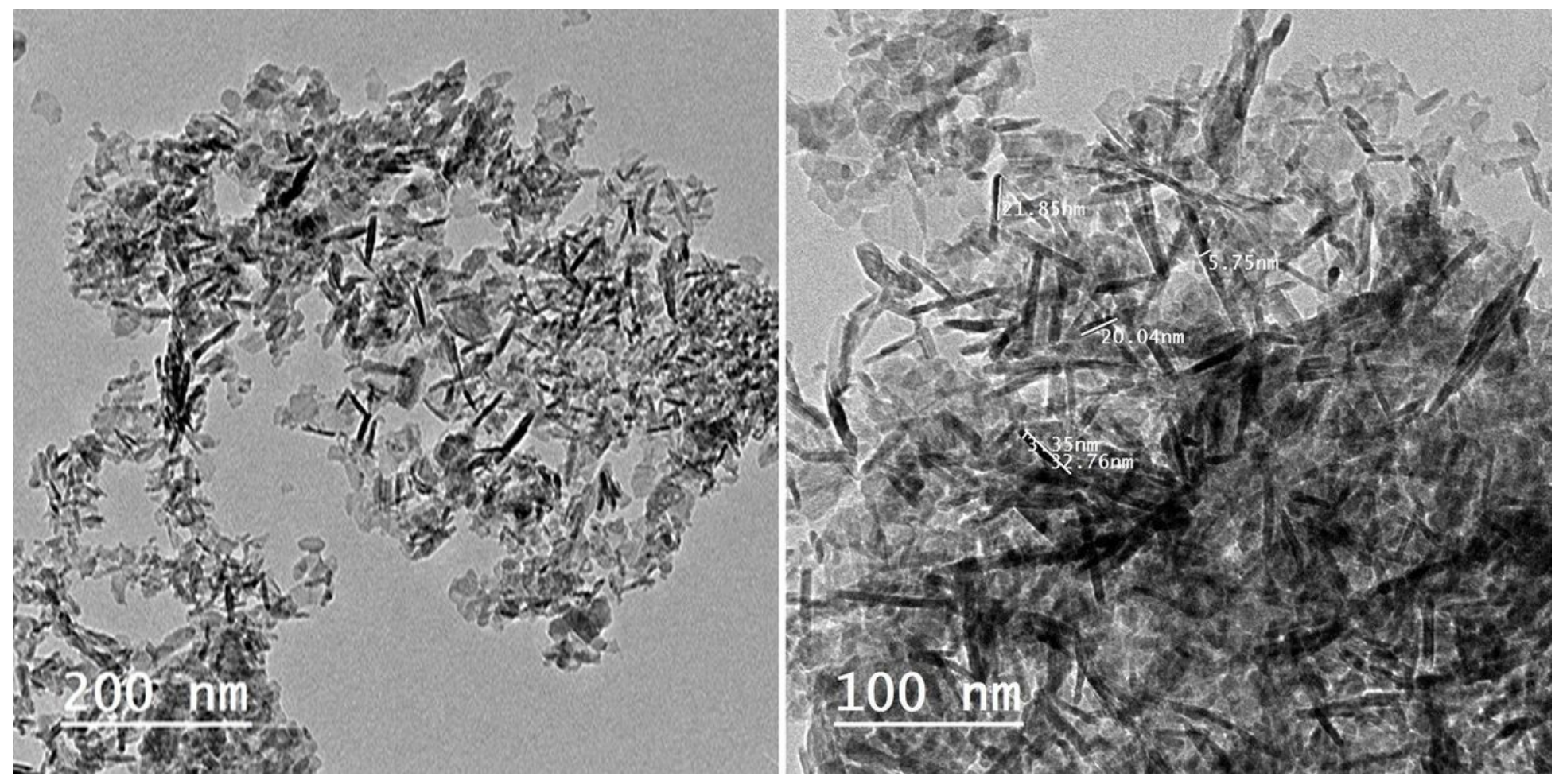

\section{Figure 1}

Transmission electron microscope image of Al203 nanoparticle in stock solutions. 
A) GST

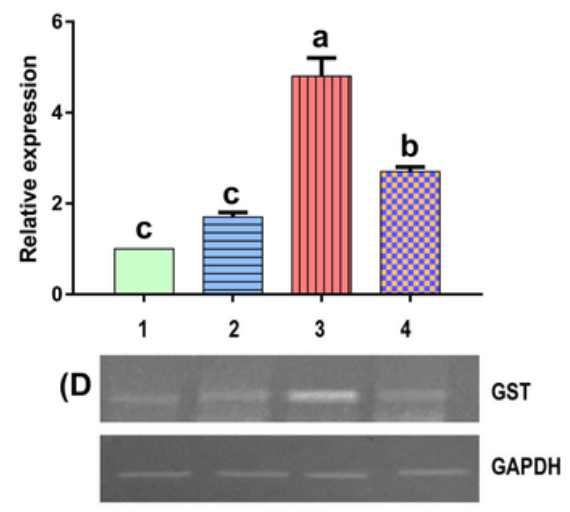

B) TNF- $\alpha$

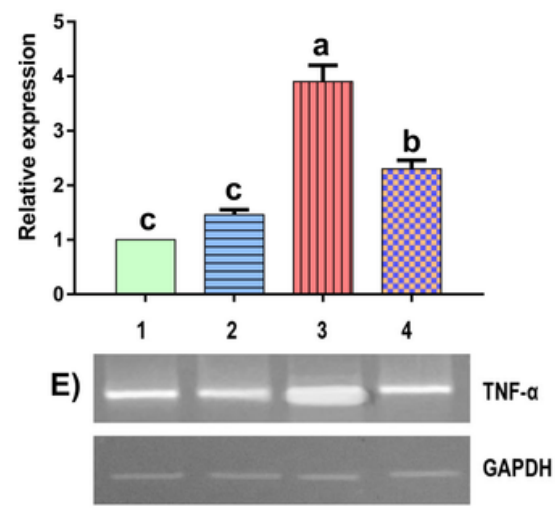

C) Caspase-3

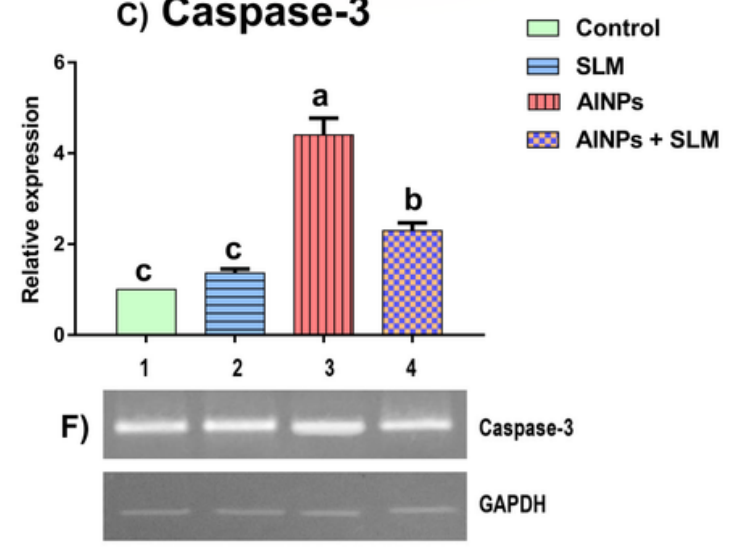

Figure 2

Effect of AINPs and/or SML on brain gene expression. Fold-change of mRNA gene expression of: A) GST, B) TNF- $a$ and C) Caspase-3 using Quantitative RT-PCR. Values are expressed as mean $\pm S E, n=5$. Bars carrying different letters $(a, b, c$, and d) are significantly different at $p \leq 0.05$. Cropped gel of electrophoretic mobility of quantitative RT-PCR products of: D) GST, E) TNF-a and F) Caspase-3 genes, with GAPDH as internal control, on 2\% agarose gel. Lane 1: control, lane 2: Sesamol, lane 3: AINPs, and lane 4: AINPs + Sesamol.
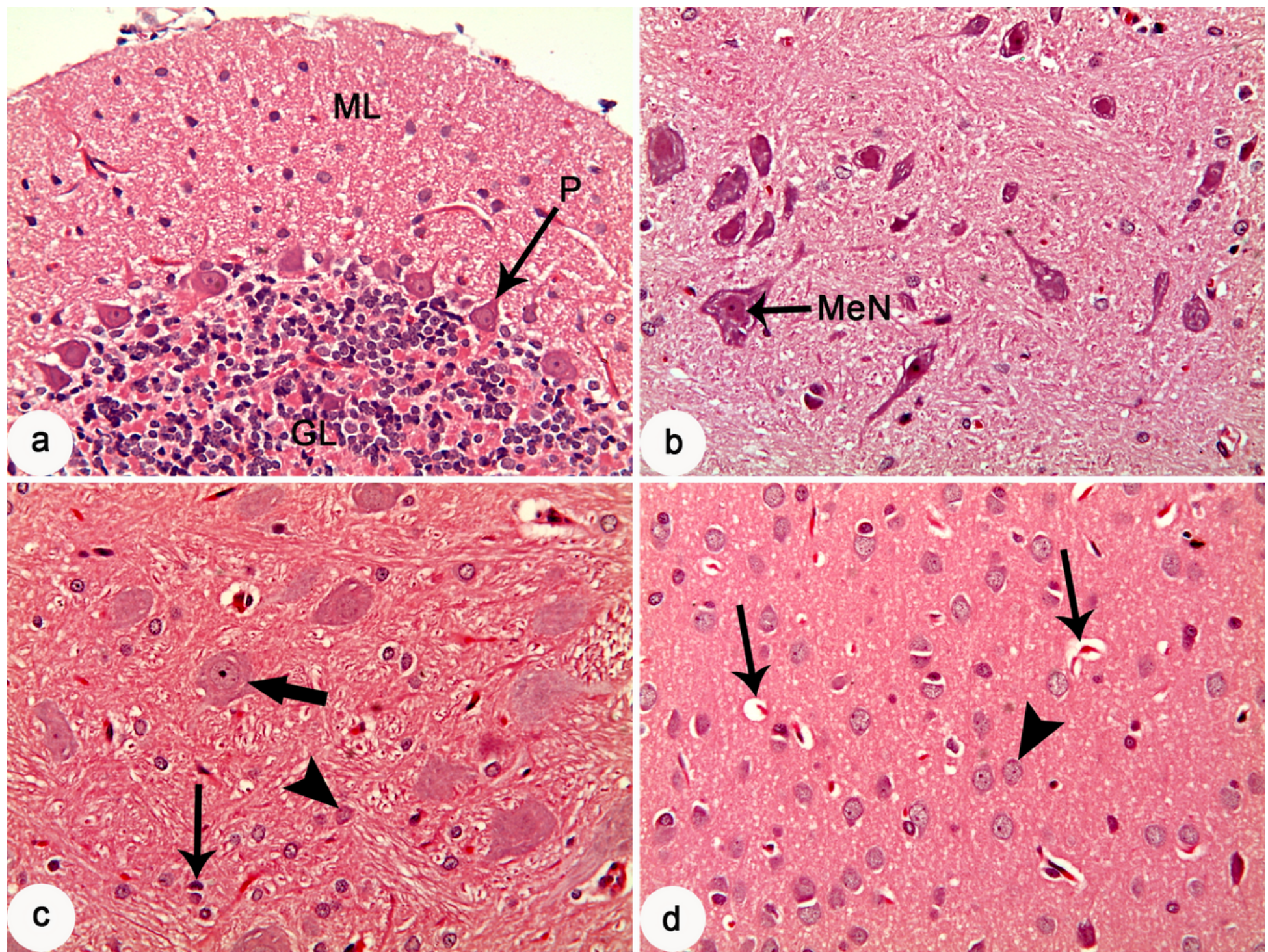


\section{Figure 3}

Brain, Rat, Sesamol (SML) treated group. a) Cerebellum: showing normal histological architectures.GL, granular layer; $\mathrm{P}$, Purkinje cell layer; ML, molecular layer. b) Medulla oblongata: showing normal histological architectures. MeN, medulla oblongata neurons. c) Midbrain red nucleus: showing normal histological architectures Motor neurons (thick arrow), Oligodendrocyte (thin arrow), Astrocyte (arrowhead). d) Cerebrum: showing normal histological architectures. Cerebral neurons (arrowhead), blood vessels (arrows). HE stain, X 20.

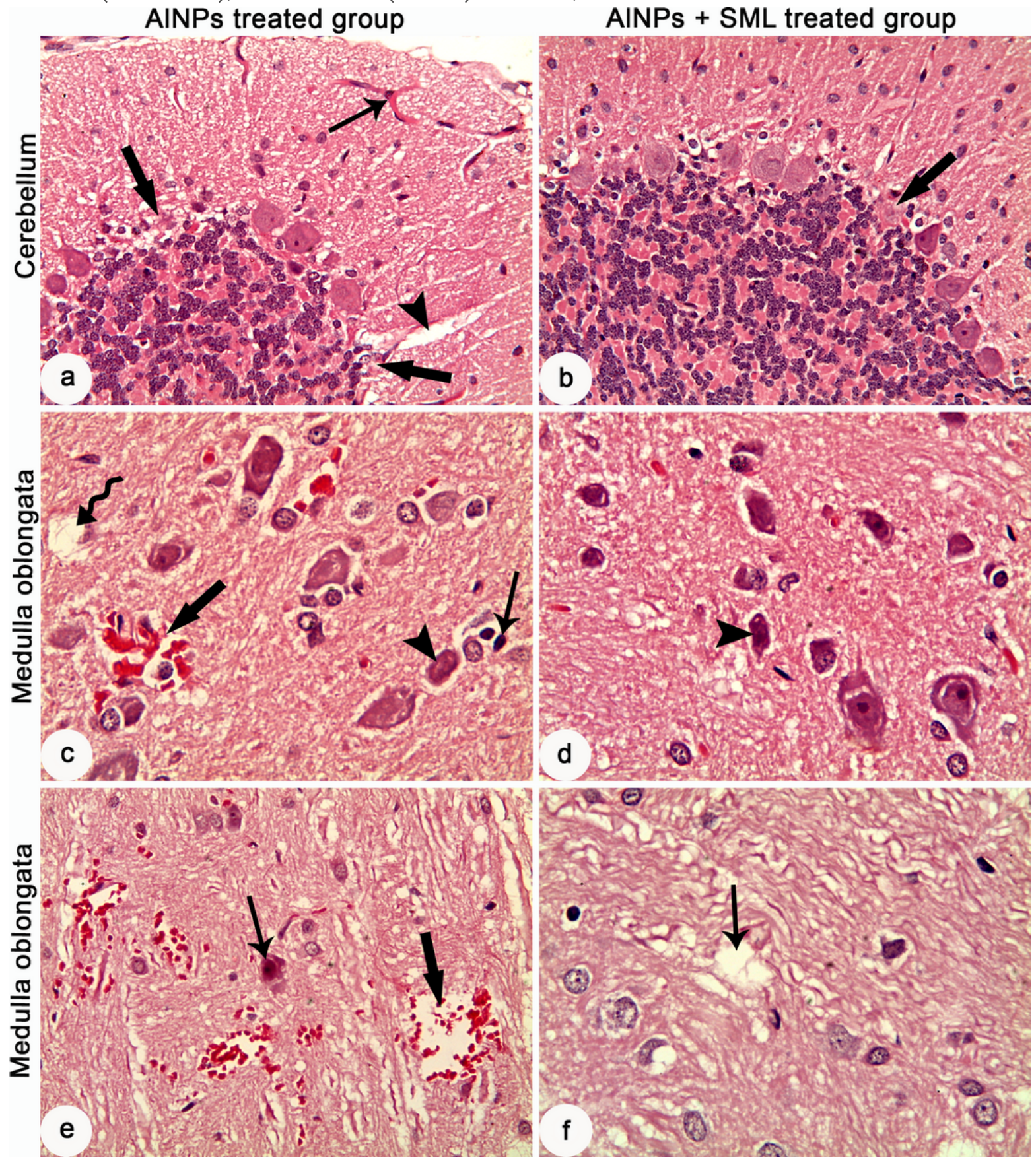

\section{Figure 4}

Brain, Rat. a, c, and e) Aluminum nanoparticles (AINPs) treated group. b, d, and f) AINPs plus Sesamol (SML) treated group. a) Cerebellum: showing necrosis and complete loss of several Purkinje cells (thick arrows), congestion (thin arrow) and edema (arrowhead) in molecular layer. b) Cerebellum: showing necrosis of a few Purkinje cells (arrow). c) Medulla oblongata: showing hemorrhage (thick arrow), edema (bended arrow), neuronal necrosis (arrowhead) and microglia activation (thin arrow). d) Medulla oblongata: showing mild neuronal necrosis (arrowhead). e) Medulla oblongata: 
showing multiple hemorrhage (thick arrow) and apoptosis on neurons (thin arrow). f) Medulla oblongata: showing edema (arrow). HE stain, X 20.
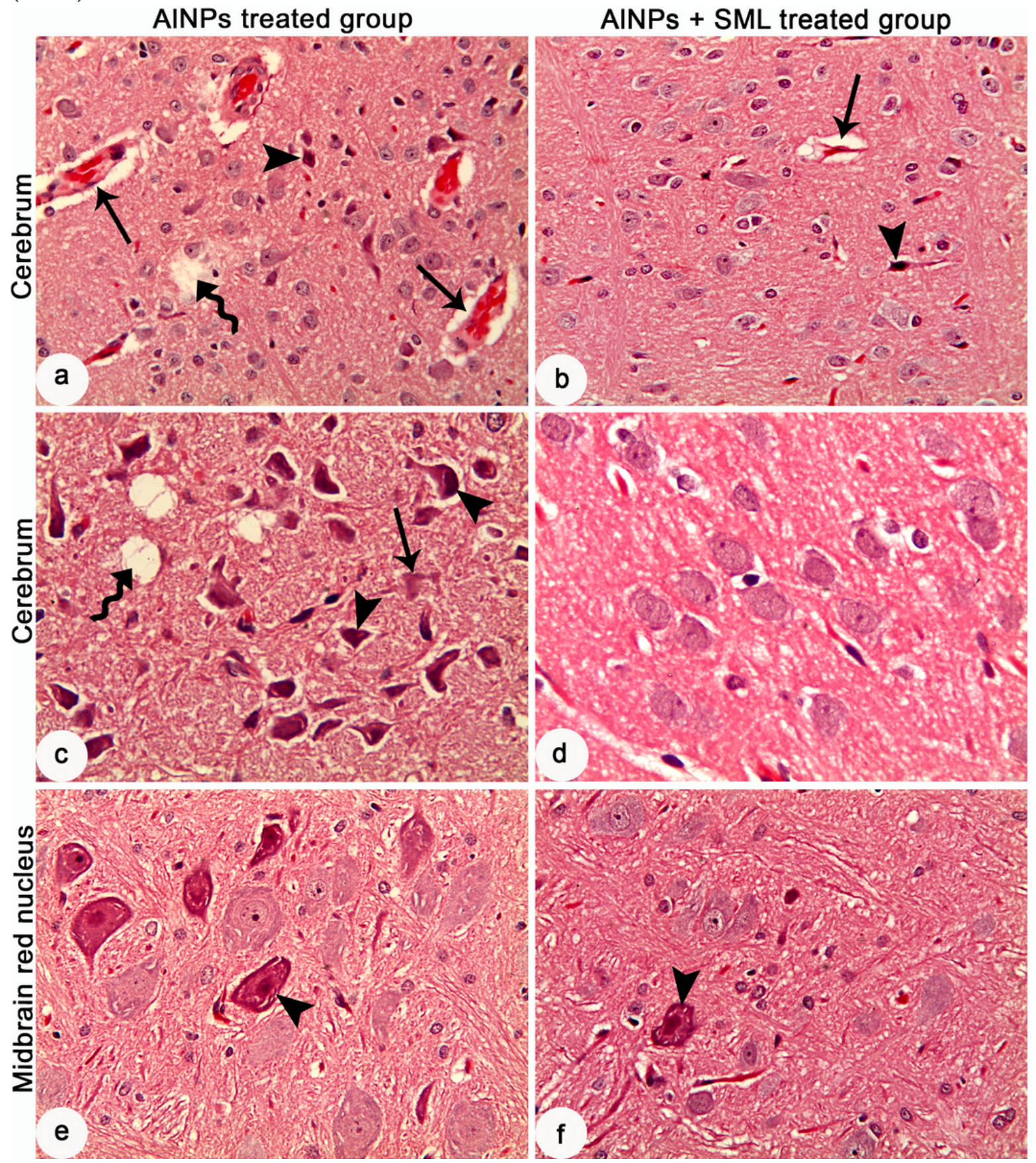

Figure 5

Brain, Rat. a, c, and e) Aluminum nanoparticles (AINPs) treated group. b, d, and f) AINPs plus Sesamol (SML) treated group. a) Cerebrum: showing a widespread perivascular edema and hyalinization of vascular walls (arrows), apoptosis of neurons (arrowhead) and edema (bended arrow). b) Cerebrum: showing a slight perivascular edema (arrow) and apoptosis of neurons (arrowhead). c) Cerebrum: showing a widespread apoptosis of neurons (arrowhead), neuronal necrosis (arrow) and edema in neuropil (bended arrow). d) Cerebrum: showing normal histological architectures. e) Midbrain red nucleus: showing neuronal degeneration of multiple neurons (arrowhead). f) Midbrain red nucleus: showing neuronal degeneration of a few neurons (arrowhead). HE stain, X 20. 